\title{
Magneto-Raman resonances in quantum wells: excitonic effects
}

\author{
L. Viña ${ }^{a, b, *}$ J.M. Calleja ${ }^{a, b}$, A. $\operatorname{Cros}^{c}$, A. Cantarero ${ }^{c}$, T.T.J.M. Berendschot ${ }^{d}$, \\ J.A.A.J. Perenboom ${ }^{d}$, K. Ploog \\ a'Inst. Ciencia de Materiales, CSIC, Campus de Cantoblanco, E-28049 Madrid, Spain

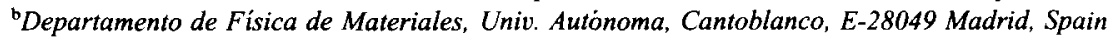 \\ 'Departamento de Física Aplicada, Univ. Valencia, E-46100 Valencia, Spain \\ ${ }^{\mathrm{d}} H F M L$, Univ. Nijmegen-Toernooiveld, NL-6525 ED Nijmegen, The Netherlands \\ 'Paul Drude Institut, Hausvogteiplatz 5, D-10117 Berlin, Germany
}

\begin{abstract}
We have measured Raman resonance profiles in GaAs/AlAs multiple quantum wells under magnetic fields up to $20 \mathrm{~T}$ for both $(100)$ and (111) crystal orientations. The results are interpreted as double Raman resonances involving magneto-excitons as intermediate states in the scattering process. A theoretical model including excitonic effects reproduces remarkably well the main features of the observed double Raman resonances. Our work clarifies the essential role of excitons in the resonant behavior of the Raman cross-section in low-dimensional semiconductors.
\end{abstract}

Light scattering by phonons is a very sensitive technique to study the electronic structure of low-dimensional semiconductors due to the resonant enhancement of the Raman signal whenever the energy of the incident(incoming resonance) or scattered-light (outgoing resonance) equals that of an electronic transition of the system. The mechanisms responsible for the scattering process are the deformation potential or, in the case of longitudinal optical (LO) phonons, the Fröhlich interaction [1]. The contribution of impurities [2] and excitonic effects [2-4] to the resonant behavior of the Raman cross-section has not been satisfactorily clarified. Excitons are expected to be relevant in two-dimensional (2D) systems due to their dominance on the optical properties [5].

A double-resonant Raman scattering (DRRS) process can occur when the energy difference between two elec-

\footnotetext{
* Correspondence address: Departamento de Física de Materiales, C-IV Universidad Autónoma de Madrid, Cantoblanco, E-28049 Madrid, Spain.
}

tronic transitions in a system equals that of a $\mathrm{LO}$ phonon. In this case the enhancement of the Raman signal will be considerably larger than that of a single resonance, due to the simultaneous vanishing of the two energy differences appearing in the denominator of the description of the Raman cross-section in third-order perturbation theory [1]. DRRS conditions have been achieved in 2D systems by a precise choice of quantum well (QW) dimensions [6] and by the application of an electric field [7]. Magnetic-field $(B)$-induced DRRS have also been observed in bulk GaAs [8] and GaAs/AlAs QWs [9]. We present new data on DRRS in GaAs/AlAs QWs induced by magnetic fields. The combination of our measurements and a calculation of the Fröhlich interaction clarifies the essential role of excitons in the resonant behavior of the Raman cross-section in 2D semiconductors.

We have investigated GaAs/AlAs multiple quantum wells (MQWs) grown by MBE on (100) and (11 1) B GaAs substrates. A first series of photoluminescence (PL), excitation (PLE) and RRS measurements 
were performed in a $13.5 \mathrm{~T}$ superconducting magnet. The second series of RRS results has been obtained at the HML of the Nijmegen University, using a $20 \mathrm{~T}$ magnet. Achromatic $\lambda / 4$ plates were used to obtain circularly polarized light and to analyze the scattered light. $B$ was applied in the Faraday configuration. A lens, placed above the sample in the high-field measurements, hindered the clear observation of selection rules due to Faraday rotation.

Fig. 1 shows the energy of the ground (solid symbols) and excited (open symbols) heavy-hole (circles) and lighthole (triangles) exciton states as a function of $\boldsymbol{B}$ obtained from PLE under $\sigma^{+}$excitation. An accurate knowledge of these energies is crucial to find the conditions for DRRS. To fit these experiments we have calculated the valence band dispersion with a $4 \times 4$ Luttinger Hamiltonian which takes into account the hh-lh mixing [10]. The conduction band has been taken to be parabolic and isotropic, with the electron mass being a function of the QW subband energy [11]. The electron in-plane effective mass $m_{x y}^{e}$ has been used as a free parameter. Excitonic effects are included through a expression for the binding energy, which takes into account $B$ and the confinement in the QW by a dimensionality parameter $D$ [12]. $D$ changes from 0.25 for a 3D exciton to 1 for a $2 \mathrm{D}$ system.

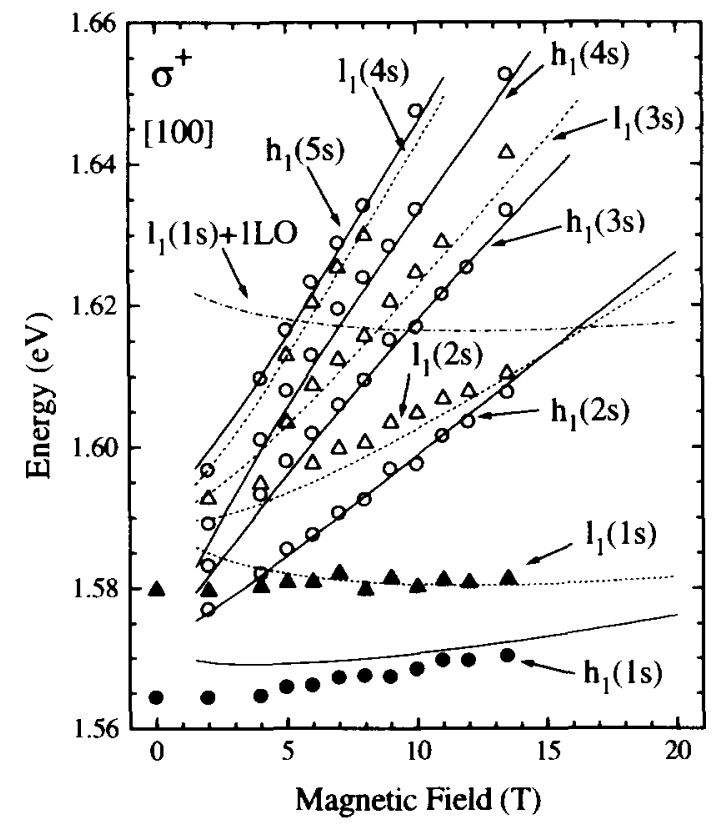

Fig. 1. Energies of the ground (solid symbols) and excited state (open symbols) heavy-hole (circles) and light-hole (triangles) excitons as a function of the magnetic field for a [100] GaAs/AIAs MQW. The lines represent the best fit of the magnetoexcitons (see text).
The solid (dashed) lines represent the best fit of the ground and excited states of hh (lh) excitons, with $D=0.6$ and $m_{x y}^{\mathrm{e}}=0.075 m_{0}$.

Raman spectra of the $(100)$ sample are shown in Fig. 2 at $18 \mathrm{~T}$ for $\sigma^{+} \sigma^{+}$polarization. The laser energies sweep the outgoing resonance condition with $l_{1}(1 \mathrm{~s})$. The $l_{1}(1 \mathrm{~s})$ $\mathrm{PL}$ is seen as a broad background underneath the sharp GaAs-LO phonon peak. One observes clearly the outgoing resonant behavior of the LO phonon as it crosses $l_{1}(1 \mathrm{~s}) . B$ corresponds to a condition of double resonance, with $l_{1}(2 \mathrm{~s})$ as incoming channel, for a laser energy of $1.619 \mathrm{eV}$. The tuning of the DRR by the field is clearly demonstrated in Fig. 3, where the laser energy has been kept at $1.619 \mathrm{eV}$ and $\boldsymbol{B}$ varied from 14.5 to $18 \mathrm{~T}$. In this field range, the outgoing resonance with $l_{1}(1 \mathrm{~s})$ is practically conserved; however the intensity of the LO-phonon diminishes markedly when $l_{1}(2 \mathrm{~s})$ is moved away from the laser energy by decreasing $\boldsymbol{B}$.

The maximum intensities of the LO-phonon, tuning the laser energy to follow an outgoing resonance with $l_{1}(1 \mathrm{~s})$, are plotted in Fig. 4 for $\sigma^{+} \sigma^{+}$(solid circles) and $\sigma^{-} \sigma^{-}$(open circles) as a function of $\boldsymbol{B}$. These constitute the DRRS profiles, which clearly show peaks at the fields corresponding to a $l_{1}(n \mathrm{~s}, n>1)-l_{1}(1 \mathrm{~s})$ energy separation equal to the LO-phonon energy. The backgrounds

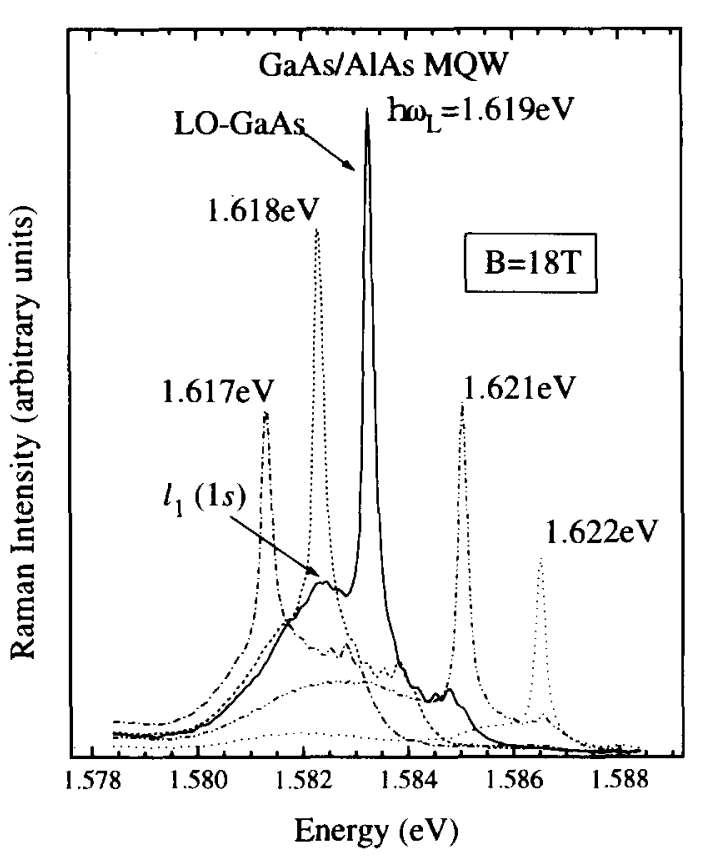

Fig. 2. Raman spectra of the sample in Fig. 1 at $18 \mathrm{~T}$ and $\sigma^{+} \sigma^{+}$ configuration for different laser energies. The sequence corresponds to a double resonant with $l_{1}(1 \mathrm{~s})$ and $l_{1}(2 \mathrm{~s})$ as outgoing and incoming channels, respectively. 


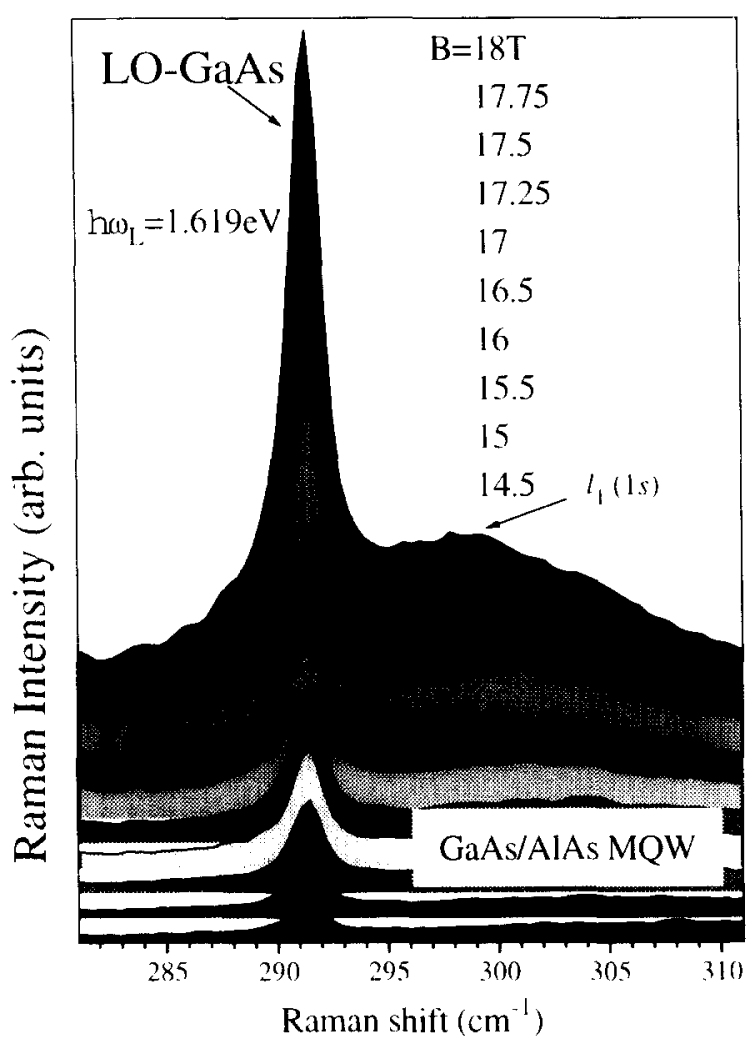

Fig. 3. Raman spectra of the sample in Fig. 1 in $\sigma^{+} \sigma^{+}$configuration for different magnetic fields and excitation at $1.619 \mathrm{eV}$. The sequence corresponds to a double resonant with $l_{1}(1 \mathrm{~s})$ and $l_{1}(2 s)$ as outgoing and incoming channels, respectively.

and the intensity ratio between the different double resonances follow a $B^{2}$ law as predicted theoretically $[8,10]$. One should emphasize that, when $l_{1}(1 \mathrm{~s})$ is selected as outgoing channel, only DRR between light-hole states are observed and that any arbitrary change in the excitonic principal quantum number $n$, which is related to the Landau quantum number, is allowed.

To calculate the Raman scattering efficiency per unit crystal length and solid angle, one has to evaluate the following expression:

$\frac{\mathrm{d} S}{\mathrm{~d} \Omega}=\frac{\omega_{\mathrm{L}} \omega_{\mathrm{S}}^{3} \eta_{\mathrm{L}} \eta_{\mathrm{S}}}{(2 \pi)^{2} c^{4}} \frac{V}{\left(\hbar \omega_{\mathrm{L}}\right)^{2}}\left|W_{\mathrm{FI}}\left(\omega_{\mathrm{S}}, e_{\mathrm{S}} ; \omega_{\mathrm{L}}, e_{\mathrm{L}}\right)\right|^{2}\left[n\left(\omega_{q}\right)+1\right]$,

where $\omega_{\mathrm{L}(\mathbf{S})}, \eta_{\mathrm{L}(\mathbf{S})}$ and $\boldsymbol{e}_{\mathrm{L}(\boldsymbol{S})}$ are the angular frequency, refractive index and polarization wave vector of the incident (L) and scattered (S) photon, and $W_{\mathbf{F I}}$ is the probability amplitude of the process, which for a one phonon emission process in third-order perturbation can be

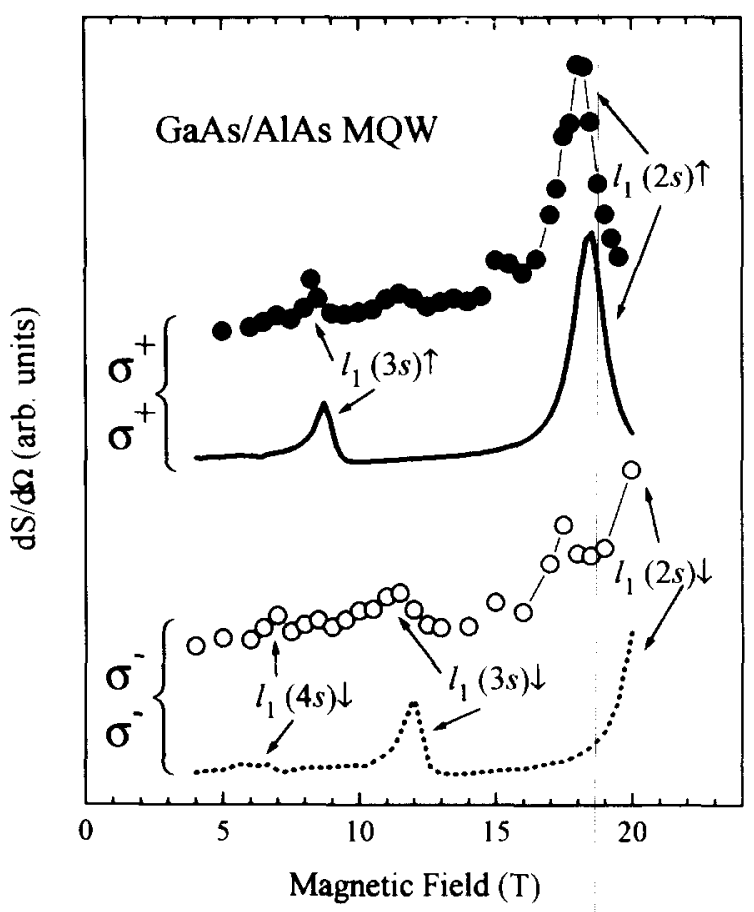

Fig. 4. Double resonant Raman profiles (symbols) for $\sigma^{+} \sigma^{+}$ (full) and $\sigma^{-} \sigma^{-}$(open) configurations. The lines represent the calculation of the Raman scattering efficiency including excitonic effects. The light-hole excitons acting as incoming channels in the double resonances are indicated.

written as

$W_{\mathrm{F} 1}=\sum_{\alpha, \beta} \frac{\left\langle 0\left|\hat{H}_{\mathrm{ER}}\right| \beta\right\rangle\left\langle\beta\left|\hat{H}_{\mathrm{EP}}\right| \alpha\right\rangle\left\langle\alpha\left|\hat{H}_{\mathrm{ER}}\right| 0\right\rangle}{\left(h \omega_{\mathrm{S}}-E_{\beta}+\mathrm{i} \Gamma_{\beta}\right)\left(h \omega_{\mathrm{L}}-E_{\alpha}+\mathrm{i} \Gamma_{\alpha}\right)}$

where $|\alpha\rangle$ and $|\beta\rangle$ are the intermediate states with energy $E_{\alpha(\beta)}$ and lifetime broadening $\Gamma_{\alpha(\beta)} . \hat{H}_{\mathrm{ER}}$ and $\hat{H}_{\mathrm{EP}}$ are the electronic-state/radiation and electronic-state/phonon interaction, respectively. If uncorrelated electron-hole pairs are considered as intermediate states, the theory predicts that the Landau quantum number is conserved and that the scattering process is intersubband, and obtains resonances whose intensities and $\boldsymbol{B}$ values are also in discrepancy with the experimental findings.

These results indicate that excitonic effects must be taken into account. The Hamiltonian of a two-dimensional magneto-exciton may be written [13] as

$$
\begin{aligned}
H_{\mathrm{exc}}= & -\frac{\hbar^{2}}{2} \frac{\partial}{\partial z_{\mathrm{e}}} \frac{1}{m_{z}^{\mathrm{e}}} \frac{\partial}{\partial z_{\mathrm{e}}}+V\left(z_{\mathrm{e}}\right)-\frac{\hbar^{2}}{2} \frac{\partial}{\partial z_{\mathrm{h}}} \frac{1}{m_{z}^{\mathrm{h}}} \frac{\partial}{\partial z_{\mathrm{h}}}+V\left(z_{\mathrm{h}}\right) \\
& -\frac{\hbar^{2}}{2 \mu_{\mathrm{x} y}^{\mathrm{h}}} \frac{1}{r} \frac{\partial}{\partial r}\left(r \frac{\partial}{\partial r}\right)-\frac{\hbar^{2}}{2 \mu_{x y}^{\mathrm{h}}} \frac{1}{r^{2}} \frac{\partial^{2}}{\partial \phi^{2}}+\frac{1}{2 i} \hbar \frac{\mu_{x y}^{\mathrm{h}}}{\gamma_{x y}^{\mathrm{h}}} \omega_{\mathrm{C}}^{\mathrm{h}} \frac{\partial}{\partial \phi} \\
& +\frac{\mu_{x y}^{\mathrm{h}}\left(w_{\mathrm{C}}^{\mathrm{h}}\right)^{2}}{8} r^{2}+V_{\mathrm{C}}\left(r, z_{\mathrm{e}}-z_{\mathrm{h}}\right) .
\end{aligned}
$$


The last term $V_{\mathrm{C}}$ is the electron-hole Coulomb interaction $\left(e^{2} / 4 \pi \varepsilon_{0} r\right)$, which we treat as a perturbation, up to second order, to the motion of the electron-hole pair in the field. We found that, in strict back-scattering configuration, the interaction exciton-photon is not able to connect excitonic states with different quantum number $n$, since they are mutually orthogonal. Thus, we must invoke a higher-order process in the Raman scattering, which we assume to be roughness scattering. In this model, the double resonances develop in triple resonances. The results of this calculation are plotted in Fig. 4 as a solid (dotted) line for the $\sigma^{+} \sigma^{+}\left(\sigma^{-} \sigma^{-}\right)$configuration. One sees clearly that the complete theory not only predicts correctly the position of the resonances as a function of $\boldsymbol{B}$ but also the relative intensities in both polarization configurations.

The experiments performed in [ $\left[\begin{array}{lll}1 & 1 & 1\end{array}\right]$ oriented samples prove that the predominant scattering mechanism is the Fröhlich interaction, since, under resonant conditions, the enhancement of the Raman signal is absent for the TO modes and is only seen for the LO modes.

In summary, we have studied magnetic-field induced DRRS in [1 000 ] and [ 1111 ]-oriented GaAs MQWs for different polarization configurations. It appears that the Raman scattering is dominated by the Fröhlich mechanism and that excitonic effects are crucial to explain the experiments. A calculation of the Raman efficiency, taking into account the Coulomb interaction between electrons and holes and roughness scattering, reproduces exceptionally well the main features of the observed double Raman resonances.
Part of this work has been financed by the European HC Mobility Programme MagNET CHRX-CT92-0062 and by the Spanish CICYT under the contract MAT-91-0201.

\section{References}

[1] See, for example, M. Cardona and G. Güntherodt (eds.), Light Scattering in Solids I to V, Topics in Applied Physics, Vol. 8, 50, 51, 54 \& 66 (Springer, Berlin).

[2] J. Menéndez, J. Lumin. 44 (1989) 285.

[3] J.E. Zucker, A. Pinczuk, D.S. Chemla, A.C. Gossard and W. Wiegmann, Phys. Rev. Lett. 51 (1983) 1293.

[4] A. Cantarero, C. Trallero-Giner and M. Cardona, Phys. Rev. B 40 (1989) 12290.

[5] L. Viña, G.E.W. Bauer, M. Potemski, J.C. Maan, E.E. Mendez and W.I. Wang, Phys. Rev. B 41 (1990) 10767.

[6] D.A. Kleinman, R.C. Miller and A.C. Gossard, Phys. Rev. B 35 (1987) 664.

[7] F. Agulló-Rueda, E.E. Mendez and J.M. Hong, Phys. Rev. B 38 (1988) 12720 .

[8] T. Ruf, R.T. Phillips, C. Trallero-Giner and M. Cardona, Phys. Rev. B 41 (1990) 3039.

[9] F. Calle, J.M. Calleja, F. Meseguer, C. Tejedor, L. Viña, C. López and K. Ploog, Phys. Rev. B 44 (1991) 1113.

[10] A. Cros, A. Cantarero, C. Trallero-Giner and M. Cardona, Phys. Rev. B 45 (1992) 6106.

[11] G.C. La Rocca and M. Cardona, Phys. Stat. Sol. (b) 167 (1991) 115.

[12] O. Akimoto and H. Hasegawa, J. Phys. Soc. Japan 22 (1967) 181.

[13] S.-R.E. Yang and L.J. Sham, Phys. Rev. Lett. 58 (1987) 2598. 